\title{
Acquirement and Interpretation of Public Documentation of Cultural Heritage —WeChat \& Post-disaster Rebuilding of Shangri-La Ancient City
}

\author{
HUO Xiaowei $^{\text {a }}$ *, ZHANG Gong ${ }^{\text {a }}$, SHANG Jin ${ }^{\text {a }}$, QI Xiaojin ${ }^{\text {a }, \text { LI Gongli }}{ }^{\text {a }}$, HUANG Zhongyuan ${ }^{\text {a }}$ \\ ${ }^{a}$ Research Centre for Historic and Cultural Cities of National Importance, Tsinghua Tongheng Urban Planning and Design Institute, \\ 100085 Beijing, China - (huoxiaowei, zhanggong, shangjin, qixiaojin, ligongli, huangzhongyuan)@thupdi.com
}

\section{Commission V, WG V/4}

KEY WORDS: Public Documentation, WeChat, Post-disaster Rebuilding, Shangri-La Ancient City

\begin{abstract}
:
The essay introduces the methodology and reflection upon the acquirement and interpretation of public documentation supporting the post-disaster reconstruction of the Shangri-La historic town after struck by fire in 2014. The proposed acquirement platform is developed on the basis of WeChat, a popular mobile communication App on smart phones in China. Historic photos and related documents of the burnt historic area are then accumulated to be analytically interpreted to inform post-disaster reconstruction planning.
\end{abstract}

\section{INTRODUCTION}

Documentation of Cultural Heritage, whether performed by professionals or the public, has its priority in preserving heritage information. Professional documentation, undertaken by trained personnel with special devices in planned processes, yields quality results that can be accessed, whereas those from laymen are whimsical and unorganized. However, sometimes the latter is advantageous in that it complements, or even replaces, the professional records, especially when what remains proves insufficient for utility purposes. Considering the popularity of cultural tourism and availability of digital devices and mobile internet, public contribution, combined with an increasing interest in heritage conservation, is playing a vital role in contemporary heritage conservation. Smart phones are proved to be effective for youth participation in heritage conservation (Poong Y.S., 2013), mobile tablets can be useful in representing perished heritage to the public (Gongli, L., 2013), and cultural heritage can be reconstructed from open access photographs (Marinos I., 2013). Such studies are brings new possibilities for cultural heritage.

\section{BACKGROUND}

On Jan, 11, 2014, the historic town of Shangri-La, where the largest Tibetan building group in China was preserved, was struck by fire that destroyed nearly one fifth of the Core Conservation Area. 343 houses around the central square and six historic streets were razed to the ground, most of which were residences and shops. Experts from Tsinghua Tongheng Urban Planning and Design Institute (THUPDI) were invited by the local government to assist in reconstruction planning.

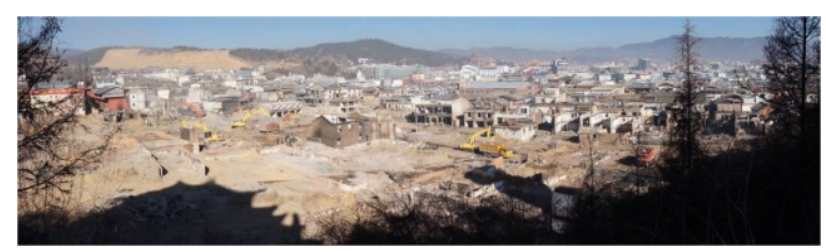

Figure 1. Dorkhar Historic Town after the Fire Photo: Dr. Ing. HUO Xiaowei

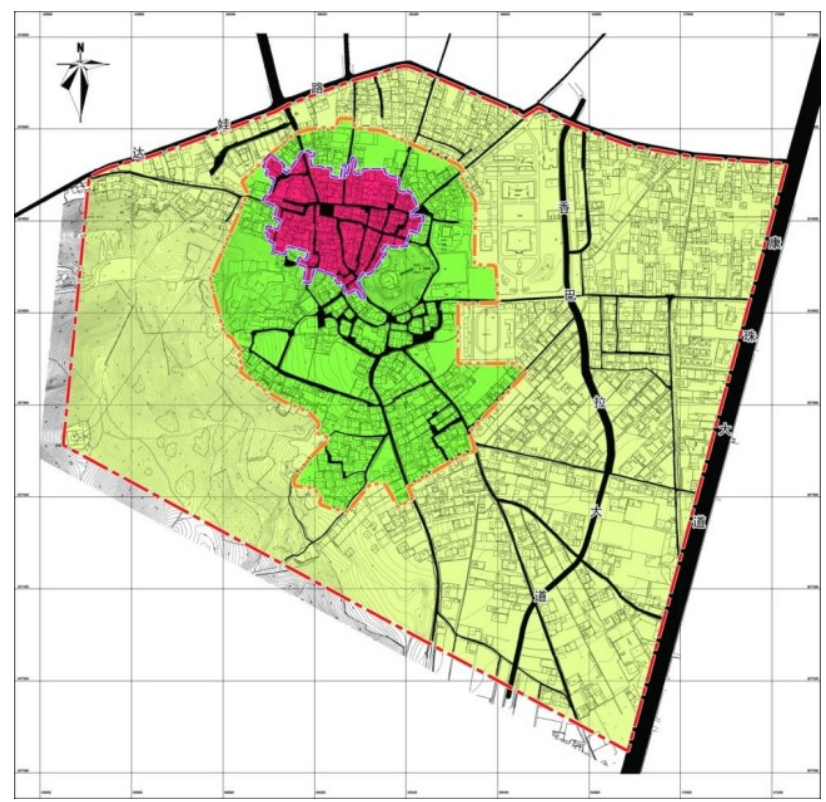

Figure 2. Fire Damaged Area in Dorkhar Historic Town

\footnotetext{
* Corresponding author.
} 


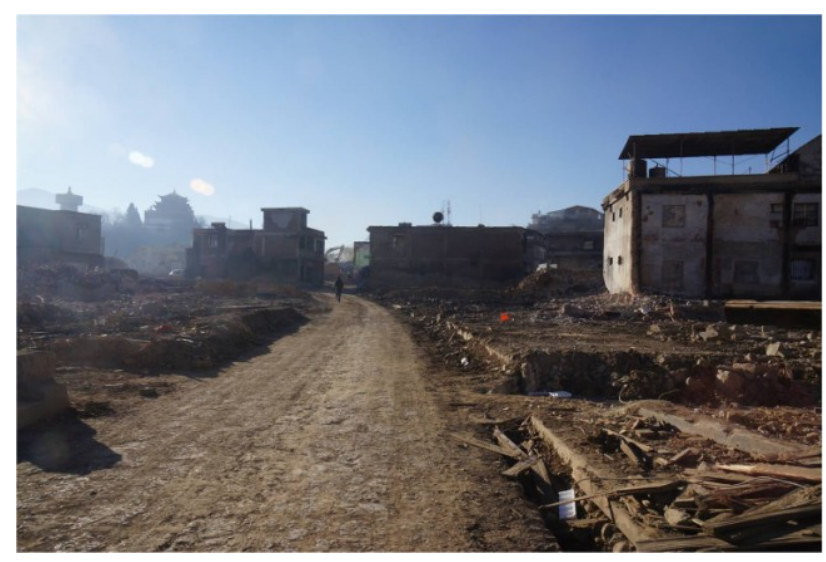

Figure 3. North Gate Street After the Fire Photo: Dr. Ing. HUO Xiaowei

Despite an urgent need to rebuild home and tourism facilities, authenticity comes in thought as a priority and necessary concern. The historic townscape, with its artistic and architectural significance, is not only what constitutes community identity but also what sustains social and economic life in Shangri-La. Unfortunately, only a few photographs, drawings, and historic maps were available, and the government demanded that reconstruction be completed by March. For this seemingly impossible task, three questions have to be answered: a) how to collect historic documents and correlate them with the physical remains as quickly as possible; b) how to identify the different time periods of collected documents of a single structure; and c) how the organized documents can inform reconstruction while preserving the authentic values, and even making the reconstruction meaningful with well-designed approaching.

\section{ESTABLISHING PUBLIC PLATFORM FOR DOCUMENT COLLECTION}

Preliminary study shows a far insufficiency in professional records of Shangri-La, and few metric and photographic archives exist which are limited to designated historic buildings. The team resorted to the public where an abundance of images could be sourced hopefully, thanks to the growth in tourists to Shangri-La from 0.52 million in 1994 to 4.27 million in 2012. The key question is how to collect and manage these documents, presumably scattered around China even the world. The answer is an effective documentation platform. The new media Social Networking Service comes to be the possible solution which is gaining greater momentum. A mobile internet-based platform could be developed for mass user contents to be concentrated and geo-referenced to inform reconstruction and future development.

\subsection{Popularity of Smart Phone and WeChat in China}

The popularity of smart phones and WeChat is fundamental to public document collection. In China, especially undeveloped areas like Shangri-La, a majority of population access the internet through a smart phone rather than a computer, and therefore mobile apps thrive. WeChat is one of the most successful free communication apps, with 468 million active users in the world since its launch in 2011. WeChat also features a Public Page with defined topic that users can subscribe. In addition to multimedia contents, the Page can facilitate interactions between its master and subscribers and surveys show that nearly $80 \%$ of the respondents are subscribers to at least one Page. ${ }^{1}$ Having these favourable conditions in mind, a Public Page of "Shangri-La Historic Photos" is created to facilitate document collecting through public participation.

\subsection{Public Sharing Platform}

In order to collect photographs of structures that existed before the fire, an interface was developed based on WeChat Public Page under Http communication protocol to facilitate the ReShangrila initiative. Functions include user messaging and interactive menu customization. Users can send heritage information to the Public Page on a smart phone, and the Page server will respond with pre-designed interactive modules to perform various tasks.

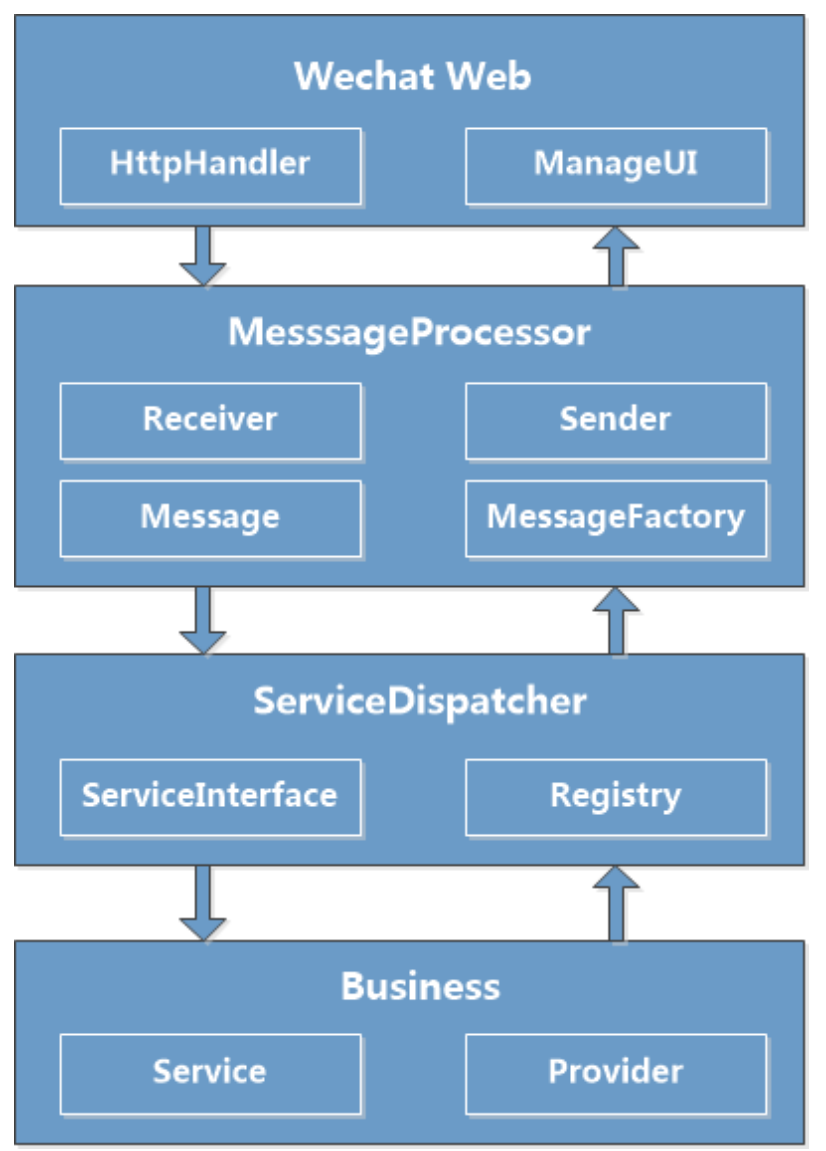

Figure 4. WeChat Mobile Service Framework

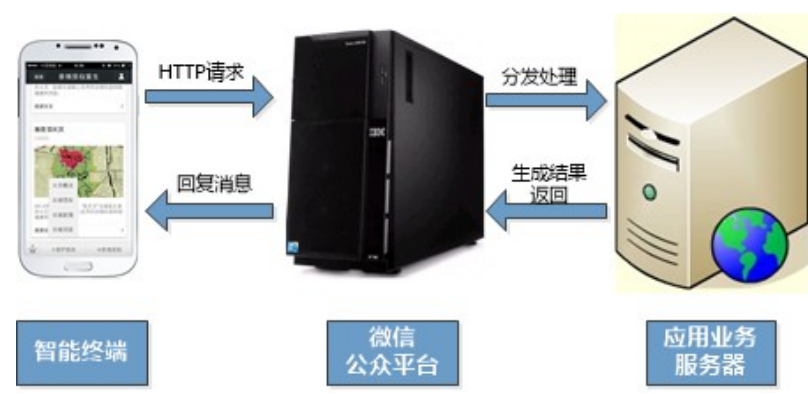

Figure 5. Mobile and Server Platform

\footnotetext{
1 Tencent Intelligence on WeChat - Statistics and Analysis, at http://tech.hexun.com/2015-01-27/172783488.html
} 


\subsection{Promotion and Dissemination}

To present the Re-Shangrila to the target groups in a shortest time, potential users are presumably categorized into Local and Remote ones according to their physical locations, and different strategies were developed.

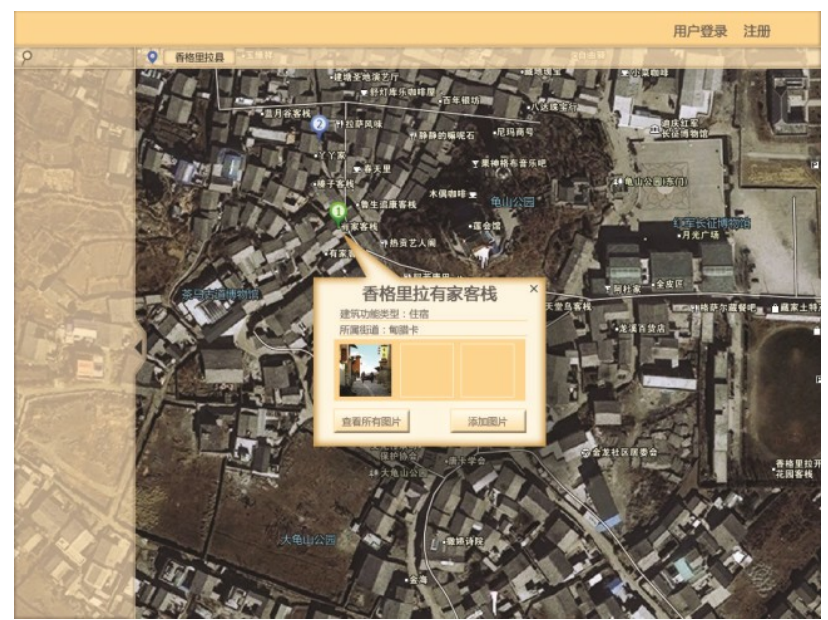

Figure 6. Re-Shangrila Interactive Map

For Local participants, the priority is to find existing local Pages with similar interests, who will potentially collaborate on promotion as partners. From web coverage of the Shangri-La fire rescue, a local grassroots cultural organization dedicated to Tibetan culture conservation and promotion called Riyuelinka came to the scene, and, after contact, agreed to produce image and text for the initiative. Circulation of generated content via Public Page and friends created a first group of Subscribers, all of whom are Tibetans. Simply by tapping their smart phones, a good amount of historic photographs were uploaded, some even dating back to as early as 20 years ago.

For Remote participants, the public document collection was organized through various media in addition to WeChat circulation, and microblogs, BBS and other traditional SNS platforms were used. Following the promotion, even previous visitors from Beijing and researchers on Shangri-La joined the Subscribers.

The combined efforts were rewarded by great numbers: 162 Subscribers contributed over 400 photographs of the historic town via WeChat, and over 30,000 microblogers read about the initiative.

\section{RECONSTRUCTION DESIGN BASED ON DOCUMENT INTERPRETATION}

Image and drawings collected from multiple sources finally enable the design team with a comprehensive set of archive, which consisted of more than 1,000 photographs and drawings, as well as pictures and survey results of the years of 1998, 2002, 2004, 2008 and 2012. There are a variety of document for almost every building in the historic streets that suffered from the fire. Of the eight heritage structures on the Provincial List of designation, seven were found to have metric survey drawings, more or less detailed. A most comprehensive documentation seemed then ready to support reconstruction design.
However, new questions arose as reconstruction design proceeded based on the collected document. First, photos were showing different periods of a same building, with different features due to historic renovation or conversion. A criterion was needed to select the one for reconstruction design. Second, the quantity and quality of photos varied from structure to structure, with a huge possession for significant built heritage, less for buildings in the historic streets, and least, if any, for ordinary residences within the quarters. So how was the collected document to inform reconstruction design and area control?

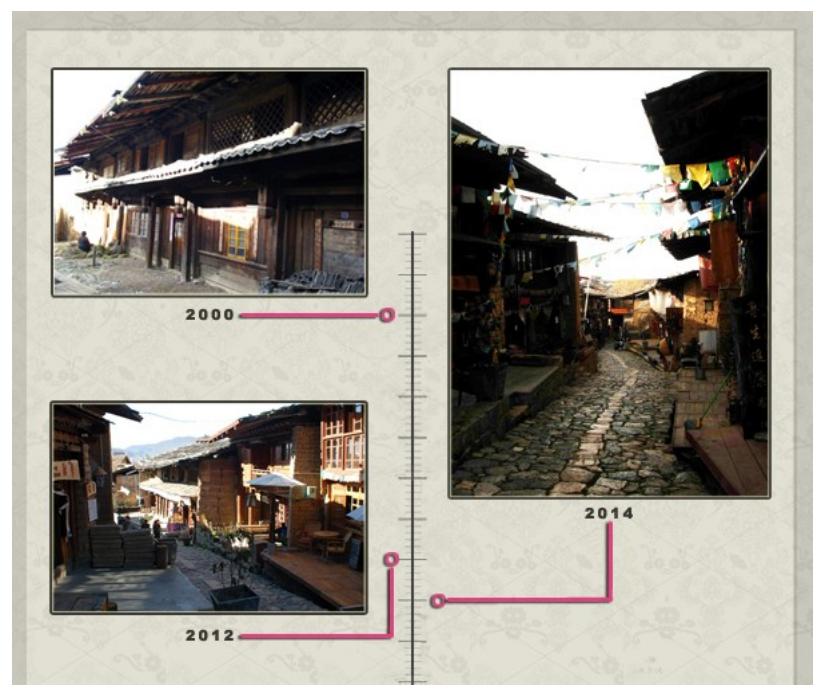

Figure 7. Collected Photo of Court Shop before the Fire

The two questions above involve the interpretation, analysis and use of the collected documents. A systematic analysis leads to the conclusion that the criteria should concentrate on the impact of streetscape changes on the traditional local character, rather than simply relying on the earliest evidence as the authentic. The traditional local character is the medium of cultural heritage value as concerns vernacular residential buildings. Reconstruction design is to pursue a maximum representation of such character from the documents of different periods. For example, a historic change in the facade without significantly altering the building itself, like a conversion of a resident into a shop in the past, is considered compatible to be restored in the reconstruction, as the local Tibetans demand.

On the other hand, the different quantity and quality of documents readily reflect the significance of the destroyed buildings. The more important it is, the more documents there are. The more details we have, the stricter appearance control there will be. For the structures with little document, it is reasonable that their reconstruction conforms to the general guidance in streetscape reconstruction which respects the organic generation of traditional building group. Local Tibetans are therefore allowed more freedom in rebuilding their homes with traditional techniques.

\subsection{Reconstruction Design of Heritage Buildings}

Repair and reconstruction of damaged heritage buildings must be cautious. As "Principles for the Conservation of Heritage Sites in China" points out in article 25,

A (heritage) building that no longer survives should not be reconstructed. Only in specially approved cases may a select 
few such former buildings be reconstructed in situ. This may occur only where there exists definite evidence that has been confirmed by experts. Reconstruction may only be undertaken after the approval process has been completed in compliance with the law and permission has been granted. Reconstructed buildings should be clearly marked as such.

It is clear from "Commentary on the Principles for the Conservation of Heritage Sites in China",

Reconstruction may be considered... When a structure has been destroyed in recent years and the public still has a strong memory and connection with it, and there exists reliable documentation. (13.3.1) Reconstruction must be based on conclusive documentary evidence; most importantly, there must also be supporting physical evidence from other sites of the same period, category, or regional origin. (13.3.3)

Survey drawings were therefore collected from local experts as solid evidences for reconstruction, while the Public Page served to gather photos showing building interior and exteriors of various periods to inform reconstruction. For example, Zhus' Shop, Court Shop, Yangs' Residence, Ka House, and Xiajugang's House were stripped of later commercial renovations in the reconstruction design to preserve their authenticity.

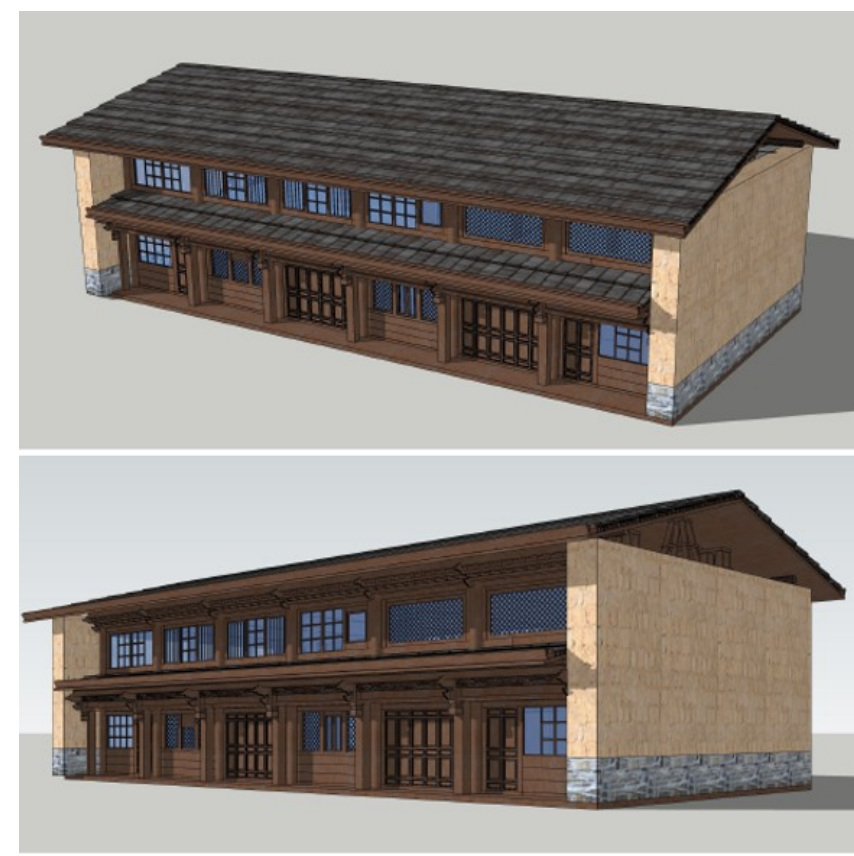

Figure 8. Reconstruction Design of the Court Shop

\subsection{Reconstruction Design of Traditional Streets}

The historic town of Shangri-La is characterized by the dominance of Tibetan Residence among harmoniously varied townscape, and the traditional streets are its shining crown jewels. However, the six traditional streets were altered in the facade for commercial purposes. Considering the significance of such features to Historic Urban Landscape, the reconstructed streets are to restore the traditional character from careful analyses while satisfying modern commercial requirements. Recognition from local residents and tenants is critical for a successful reconstruction design.
Keeping the above consideration in mind, the project team identified the traditional Tibetan architectural features from each building on the street by examining the collected images of different periods. A "benchmark year" was then used for reconstruction where its height, relation to adjacent buildings, ground level, bay width, facade features, windows and doors were clearly specified. With these as evidences for subsequent planning control, inadequate transformation and expansion to traditional buildings were removed which were restored according to the historic images. Yet the windows and doors were designed to meet contemporary needs.

\subsection{Reconstruction Guideline and Traditional Residence Handbook}

Because reconstruction followed the "Uniform Planning and Self-Build" model, it was impossible, and unnecessary to produce one blueprint for all the residents without considering individual needs in such a short time. The team therefore proposed three types of Instructions/Code based on Land Use, Building, and Residence Construction, which can be applied to planning in a flexible manner.

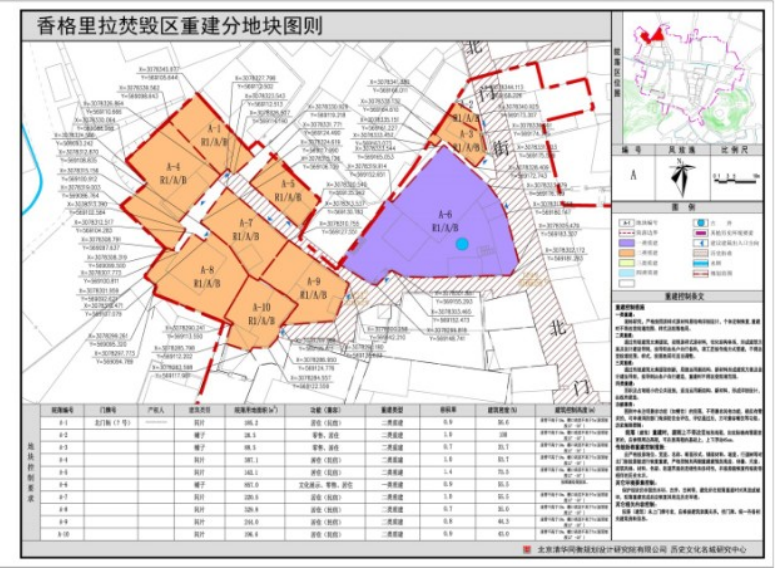

Figure 9. Land Use Instructions

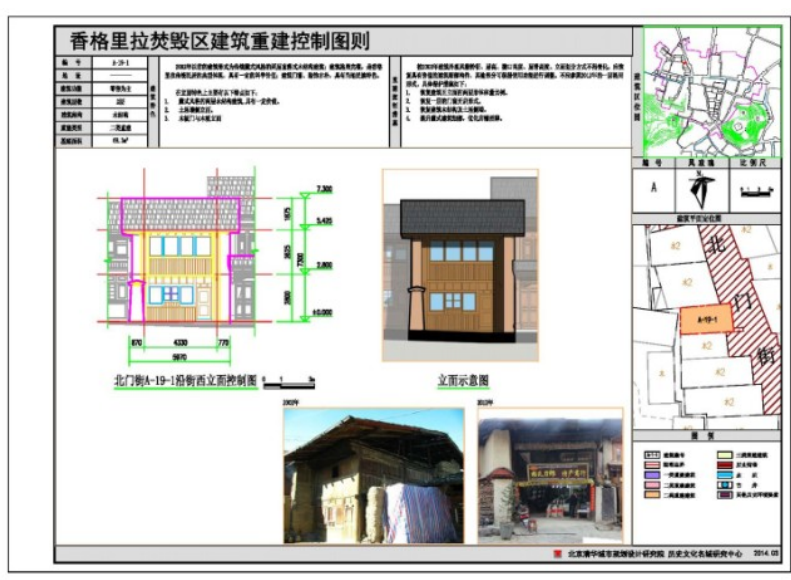

Figure 10. Building Instructions

The huge amount of collected images are important evidences in the Building Instruction for architectural characterization and facade control in addition to visual references. In the Residence Construction Instruction, the images were also used to summarize the traditional characters as well as construction techniques in Shangri-La, which were then composed into an 
illustrated handbook for construction. This handbook also provided models for the modernization of traditional residences and technically informed the locals while preserving the architectural diversity in the historic town.

\section{EXPERIENCES}

Successful experiences from the data collection for the postdisaster reconstruction of Shangri-La lend itself to similar heritage risk preparedness and documentations.

A) Multiple technological means should be applied to the regular monitoring of heritage assets. The case of Dorkhar has highlighted the importance of heritage documentation prior to the unexpected fire. For settlement heritage, the local culture, layout and fabric, boundary, architectural style and tectonics, as well as decorative features should be included in the heritage inventory and updated on a regular basis.

B) Emergency call of data collection must be established immediately after the disaster has been controlled or mitigated, where an extensive social network plays a critical role in that:

i. The building and settlement are constantly changing to human life and the chronicle information as the authentic documentation of different times must be recorded to reflect their truthful development, which will support identification of critical alteration whenever necessary.

ii. Heritage documentation can benefit from technology inspired crowd sourcing, which will capture more details than a single professional inventory, especially when the heritage asset is lost to unexpected disaster.

iii. The collectively contributed heritage documentation is itself the memory of a group connected to the asset via both virtual and physical bonds. Such documentation creates a common identity that has psychological and emotional implications.

C) Extensive use of social network by local residents, mobile internet in particular, will gain support from the local community so as to enhance the efficiency and extensiveness in public participation.

\section{CONCLUSION AND REFLECTIONS}

Loss of some heritages due to sudden strike of disasters is inevitable for humanity. A mere sight of what has survived over hundreds or thousands of years reminds everyone of the harsh tear and wear. It is such vicissitude that makes our heritage invaluable. Although disputed and even criticized, post-disaster reconstruction is nothing less than a remedy to prevent total loss. But, without sufficient documentation, even such a rescue is doomed to be non-possibility. Public documentation shows the gleaming beam of hope. Discreet documents of various sources can be efficiently collected and managed, which, after analysis and interpretation, enhances reconstruction in an unprecedented manner.

The acquirement and interpretation of public document to inform Shangri-La reconstruction has yielded valuable experiences:

I. Public documentation of cultural heritage is more than feasible by means of Social Networking like WeChat on smart phones. Such communicative measure readily lends itself to an extensive public documentation system that transcends territorial boundaries. The efficiency in dissemination and data retrieval as well as user interaction is unfathomable.

II. Public documentation by means of new media, enhanced by promotion via traditional media like television and newspaper, ignites extensive simultaneous social participation, which transforms heritage reconstruction into a literal social event. Augmented with mass documents that inform reconstruction design, the restored heritage is imbued with new cultural and social significance.

III. Public documentation can inform heritage reconstruction design. For this case in Shangri-La, it was fortunate to acquire detailed documents of the historic town before the fire from special sources later, which was used as the major evidence for reconstruction design, and those from the public contributed as references. Yet there is tremendous potential in public documentation which will complement the professional in the process of reconstruction design. The faith in this promising approach is shedding light beyond the reaches of the project team.

IV. Open and easy access of WeChat and smart phones is seamlessly bridged to various media like microblog and website. The versatility of WeChat is bringing more possibility than public documentation. For one, acquired image, when identified and georefenced, will be marked on the interactive map in combination with texts and coordinates, hence a complete open resource of the memory and historic information of the historic town. This source can be used to virtual cultural tourism or heritage monitoring. Another feature, the Public Page, allows dissemination of the knowledge and value of the settlement heritage in Shangri-La to all Subscribers, who can also send enquiries about fire damage and reconstruction planning. The website information and acknowledgement to all contributors were also broadcast on the Public Page.

V. Success of the WeChat operation depends on the careful design of user interaction and functions. Immediate response to participants is critical to promoting public participation. ReShangrila is therefore a first pilot initiative to public documentation for informed heritage reconstruction, and more innovations will build up a bright future for heritage conservation in the world.

\section{ACKNOWLEDGEMENTS}

All team members of the Re-Shangrila initiative deserve sincere gratitude because of their passion and devotion. Under the supervision of Dr. Ing. HUO Xiaowei, the architectural conservation group of ZHANG Gong, CHEN Jie, DU Fang, HUANG Haoyan, WANG Hecai, XU Huijun, and ZHENG Xin, aided by the research group of QI Xiaojin, WU Qilin, and LUO Wen, as well as the technical specialist of LI Gongli, HUANG Zhongyuan, LIN Liubin, CHEN Huilian and CHEN Jiepin, all of whom come from Tsinghua Tongheng Urban Planning and Design Institute in Beijing, collaborated with ZHAI Hui and ZHANG Xinyan from Faculty of Civil Engineering and Architecture, Kunming University of Science and Technology in creating an innovative reconstruction methodology to bring back the cherished heritage in Shangri-La. 


\section{REFERENCES}

HUO Xiaowei, 2014. Traditional Village Conservation and Wrath of the Fire God - Investigation and Reflection of the Dorkhar Historic Town after the Fire Disaster, In: China Culture, April 17, 2014

Marinos, I., 2013. Online 4D Reconstruction Using MultiImages Available Under Open Access. In: ISPRS Annals of the Photogrammetry, Remote Sensing and Spatial Information Sciences, Volume II-5/W1, 2013

Poong Y.S., 2013. Possibility to use mobile learning to promote world heritage site preservation awareness in luang prabang, Lao PDR: A Readiness Study. In: 2013XXIV International CIPA Symposium, (Strasbourg, France 2 - 6 September 2013.)

Silberman, N., 2012. Collective memory as affirmation: peoplecentered cultural heritage in a digital age. In: Heritage and Social Media: Understanding Heritage in a Participatory Culture. New York, pp.13-30.

Gongli, L. et al., 2013. A Mobile Application for Virtual Heritage and UGC Public Sharing, In: 2013XXIV International CIPA Symposium, (Strasbourg, France 2 - 6 September 2013.) 